Article

\title{
Adaptive Wavelet Threshold Denoising Method for Machinery Sound Based on Improved Fruit Fly Optimization Algorithm
}

\author{
Jing Xu ${ }^{1}$, Zhongbin Wang ${ }^{1, *}$, Chao Tan ${ }^{1}$, Lei Si ${ }^{1,2}$, Lin Zhang ${ }^{1,3}$ and Xinhua Liu ${ }^{1}$ \\ 1 School of Mechatronic Engineering, China University of Mining and Technology, No.1 Daxue Road, \\ Xuzhou 221116, China; xujingcmee@cumt.edu.cn (J.X.); tccadcumt@126.com (C.T.); sileicool@163.com (L.S.); \\ lin.zhang_2014@hotmail.com (L.Z.); 1_xinhua_2006@126.com (X.L.) \\ 2 School of Information and Electrical Engineering, China University of Mining and Technology, \\ No.1 Daxue Road, Xuzhou 221116, China \\ 3 Institute for Neural Computation, University of California, San Diego (UCSD), No.3950 Mahaila Ave, \\ San Diego, CA 92093, USA \\ * Correspondence: wangzbpaper@126.com; Tel./Fax: +86-516-8388-4512 \\ Academic Editor: Gino Iannace \\ Received: 5 May 2016; Accepted: 1 July 2016; Published: 6 July 2016
}

\begin{abstract}
As the sound signal of a machine contains abundant information and is easy to measure, acoustic-based monitoring or diagnosis systems exhibit obvious superiority, especially in some extreme conditions. However, the sound directly collected from industrial field is always polluted. In order to eliminate noise components from machinery sound, a wavelet threshold denoising method optimized by an improved fruit fly optimization algorithm (WTD-IFOA) is proposed in this paper. The sound is firstly decomposed by wavelet transform (WT) to obtain coefficients of each level. As the wavelet threshold functions proposed by Donoho were discontinuous, many modified functions with continuous first and second order derivative were presented to realize adaptively denoising. However, the function-based denoising process is time-consuming and it is difficult to find optimal thresholds. To overcome these problems, fruit fly optimization algorithm (FOA) was introduced to the process. Moreover, to avoid falling into local extremes, an improved fly distance range obeying normal distribution was proposed on the basis of original FOA. Then, sound signal of a motor was recorded in a soundproof laboratory, and Gauss white noise was added into the signal. The simulation results illustrated the effectiveness and superiority of the proposed approach by a comprehensive comparison among five typical methods. Finally, an industrial application on a shearer in coal mining working face was performed to demonstrate the practical effect.
\end{abstract}

Keywords: wavelet threshold denoising; sound signal; wavelet transform; improved fruit fly optimization algorithm; fly distance range

\section{Introduction}

Generally, the vibration and strain signals of a machine are mostly applied to provide dynamic information of the machine's working condition [1,2], even though they have some common disadvantages, such as contact measurement, limited detecting positions and difficult to maintain detectors in some severe situations. Therefore, vibration and strain measuring is inappropriate or sometimes even impossible in these cases. On the other hand, the sound signal of a machine can be a significant criterion for state recognition or fault diagnosis because it is convenient to collectand does not affect the machine [3]. Thus, acoustic-based diagnosis (ABS) has received much attention in recent years [4]. One of the most important preconditions for ABS is eliminating noise from the initial sound signal, and the performance of denoising directly influences the effect of subsequent processing $[5,6]$. 
Preliminary analysis of machinery sound shows that significant details distribute in both time and frequency domains, which implies that noise elimination methods considering both scales would perform better than those only focusing one. Throughout the development of signal processing, the most influential time-frequency joint analysis approaches are Fast Fourier Transform (FFT) and Wavelet Transform (WT) [7]. WT was firstly proposed by Mallat in 1989 [8]. As the window function of FFT is fixed, WT is obviously superior to FFT for non-stationary signal as the property of characterizing local features in both domains. Six years later, Donoho proposed hard-threshold and soft-threshold denoising solutions based on WT. The corresponding threshold value was selected by combining WT and Stein's unbiased risk estimate (SURE) [9]. However, since the derivative of standard threshold function is not continuous and lacks adaptability, many improved wavelet noise reduction methods have been proposed [10-12]. With the extensive application of artificial intelligence in recent years, adaptive threshold selecting approaches based on intelligent optimization algorithms, such as the particle swarm optimization (PSO), genetic algorithm (GA) and ant colony optimization (ACO) have been adopted gradually [13-15].

Fruit fly optimization algorithm (FOA) was proposed by Pan in 2012 [16-18]. As a meta-heuristic method, FOA simulates the intelligent foraging behavior of fruit fly group in food finding process [19]. The fruit fly is superior to other species with regard to its senses of osphresis and vision, it can even a smell food source from $40 \mathrm{~km}$ away and locate other flocks by its sensitive vision [20]. The FOA has many advantages compared with the above optimization algorithms, such as simple structure, immediately accessible for practical applications, ease of implementation and rapid convergence rate. Since the FOA was proposed, it has been widely applied in financial parameter optimization [21], forecasting [22], scheduling [23], etc. However, like other optimization algorithms, the basic FOA also has the possibility of falling into local extremes due to its fixed fly distance range [24].

Bearing the above observations in mind, an adaptive wavelet threshold denoising method for machinery sound based on an improved FOA (WTD-IFOA) is proposed. The rest of this paper is organized as follows. In Section 2, some related works are outlined based on the literature. In Section 3 , the basic wavelet noise elimination method and optimization process of FOA are presented. In Section 4, an improved fly distance range, obeying normal distribution, is performed, and the denoising solution based on WTD-IFOA is elaborated. In Section 5, Gauss white noise is added into the motor sound signal to verify the effectiveness and superiority of the proposed method, and an industrial application is performed. Some conclusions and outlooks are summarized in Section 6.

\section{Literature Review}

Recent publications relevant to this paper are mainly concerned with two research streams: wavelet threshold denoising and fruit fly optimization algorithm. In this section, we try to summarize the relevant literature.

\subsection{Wavelet Threshold Denoising}

Traditional denoising methods, such as low-pass filter, Kalman filter and median filter, aim either at the time domain or the frequency domain. However, single-scale representations of signals are often inadequate when attempting to separate signals from noisy data. By combining the two scales, wavelet threshold denoising presents obvious superiority. According to the wavelet threshold denoising theory proposed by Donoho, the optimal threshold should diminish the noise but preserve the signal as much as possible [25]. The traditional hard-threshold function exhibits some discontinuities and may be unstable or more sensitive to small changes in the data, while in soft thresholding the wavelet coefficients are reduced by a quantity equal to the threshold value, which will induce the deviation when the filtered wavelet coefficients is reconstructed [26]. Moreover, the threshold is fixed once determined and adaptability is weak during the denoising process. In order to overcome the disadvantages of the original threshold functions proposed by Donoho, many adaptive denoising approaches have been elaborated by researchers. Improved solutions can be divided into two streams: 
the first on the improvements of threshold function and the other focus on searching optimal threshold through intelligent algorithms. The threshold function-based methods aim at establishing appropriate function with continuous derivative and selecting thresholds based on gradient descent algorithm. In [10], a new adaptive denoising function with continuous first and second order derivative is presented based on SURE model. In [27], an adaptive logarithmic wavelet threshold denoising function was proposed to select optimal threshold for each decomposition level. Relative to hard and soft functions, the proposed approach increased the signal-to-noise ratio by $44.2 \%$ and $27.9 \%$, and decreased processing time by $37.6 \%$ and $38.5 \%$, respectively. With the rapid development of artificial intelligent optimization algorithm, intelligent searching-based wavelet denoising approaches have been widely applied in recent years. In [14], a PSO-based image denoising method was proposed for learning the parameters of the adaptive thresholding function required for optimum performance. Li et al. adopted an adaptive denoising solution for partial discharge signals based on threshold function and genetic algorithm (GA), and the result presents significantly smaller waveform distortion and magnitude errors than the Donoho's soft threshold estimation [15]. In order to eliminate noise components of satellite images, some stochastic global optimization techniques such as Cuckoo Search (CS) algorithm, artificial bee colony (ABC), and PSO as well as their different variants have been exploited for learning the parameters of adaptive thresholding function in [28].

\subsection{Fruit Fly Optimization Algorithm}

Although it is not long since FOA was put forward, it has aroused much attention and scored great academic achievements. In [21], FOA was adopted to optimize general regression neural network, and the simulation result showed the superiority compared with other intelligent optimization algorithms. In [29], an annual electric load forecasting method was proposed by the least squares support vector machine (LSSVM) model. The FOA was used to determine appropriate parameters of the model, and an experiment, with the mean absolute percentage error of $1.305 \%$, proved the validity of the approach. Although the FOA has an extensive application in many fields, there still exists the possibility of getting into the local extreme [24]. The main reason lies in the fruit fly individuals move toward fixed fly distance range in the iteration of optimization. Once the fruit fly group fall into the local extreme and the fly distance range is not big enough, the optimization process is prone to fail [30]. On the other hand, excessive fly distance range may lead to slow convergence rate of the iteration process. In [23], an improved FOA was presented to solve the joint replenishment problems. In order to avoid local optimal solution, swarm collaboration and random perturbation were added into original FOA. Pan et al. presented a changeable fly distance range in FOA to eliminate the drawbacks lies with fixed values of search radius, and 29 benchmark functions were carried out to make a comparison with basic FOA [24]. Yuan et al. proposed a multi-swarm FOA, where several sub-swarms moving independently in the search space with the aim of simultaneously exploring global optimal and local behavior between sub-swarms is also considered [31]. In [32], an improved FOA, called linear generation mechanism of candidate solution fruit fly optimization algorithm (LGMS-FOA), was introduced for solving optimization problems. Four disadvantages of the original FOA were listed and some improvements were operated, and the simulation result showed local extreme could be avoided efficiently.

\subsection{Discussion}

Many valuable wavelet denoising methods have been proposed and applied by researchers in recent decades, which greatly pushes forward the development of this field. However, there are still some shortcomings including the following. Firstly, the disadvantage of weak adaptability seriously restricted the development of Donoho's wavelet threshold approaches. Secondly, adaptive noise elimination methods based on gradient descent algorithm were also limited because of the great amount of calculation. The above approaches were gradually replaced by intelligent optimization-based algorithms. Thirdly, the iterative process of the common optimization solution has the problems of slow convergence rate and high complexity of coding. In [15], the proposed method 
based on GA adaptive threshold cost more than 38.66 times the calculation time compared to soft threshold approach. Moreover, the FOA has great advantages in iteration rate and encoding efficiency, but still has the probability of falling into local extreme. Many improvements have been elaborated by past scholars, but few researchers could balance both local extreme and iterative rate.

Therefore, a novel wavelet threshold denoising method optimized by an improved FOA is proposed in this paper. The fly distance range obeying uniform distribution in the basic FOA is replaced by the following normal distribution. Both local extreme and iterative rate are taken into consideration. A series of simulations and an industrial application prove the effectiveness and superiority of the proposed method.

\section{Basic Theory}

\subsection{Wavelet Threshold Denoising}

Fundamental theory of wavelet denoising can be concluded as follows: wavelet decomposition is firstly conducted on the noisy signal, then wavelet coefficients that belong to useful signal are kept and others are eliminated, and finally inverse wavelet transform is operated to reconstruct the remainder coefficients.

Assume that the noisy signal series $x=\left\{x_{1}, x_{2}, x_{3}, \ldots, x_{k}\right\}$ can be expressed as follows:

$$
x_{i}=s_{i}+n_{i}
$$

where $i=1,2,3, \ldots, k, s=\left\{s_{1}, s_{2}, s_{3}, \ldots, s_{k}\right\}$ is the useful initial signal and $n=\left\{n_{1}, n_{2}, s_{3}, \ldots, n_{k}\right\}$ is noise signal.

Then $x$ is decomposed by $J$ levels WT and the $i$-th wavelet coefficient in $j$-th can be presented as $d_{i, j}$, where $j=1,2,3, \ldots, J$. Since WT is a kind of linear transform, wavelet coefficients of $x$ are consisted of ones decomposed by $s$, denoted as $U_{i, j}$, and that of $n$, called $V_{i, j}$. The purpose of wavelet denoising is to eliminate $V_{i, j}$ and obtain the estimate signal $\hat{s}$ of the noisy signal. The ideal $\hat{s}$ has a minimum mean square error with $s$ under the premise of eliminating noise component furthest. The mean square error (MSE) $\xi$ can be calculated as follows:

$$
\xi[\hat{s}, s]=\frac{1}{k}|| \hat{s}-s||=\frac{1}{k} \sum_{i=1}^{k}\left[\hat{s}_{i}-s_{i}\right]^{2}
$$

The threshold during the denoising process is calculated according to SURE model, which can be obtained as follows:

$$
\lambda_{j}=\operatorname{MAD}\left(\left|d_{i, j}\right|\right) / q
$$

where $\lambda_{j}$ denotes the threshold of $j$-th level, $\operatorname{MAD}(\cdot)$ is a median value function and the value range of $q$ is $[0.4,1]$ in general.

There are two typical threshold functions during the wavelet denoising process, the first called hard-threshold:

$$
\hat{d}_{i, j}=\left\{\begin{array}{rc}
d_{i, j}, & \text { for }\left|d_{i, j}\right| \geqslant \lambda_{j} \\
0, & \text { otherwise }
\end{array}\right.
$$

where $\hat{t}_{i, j}$ donates the wavelet coefficient of denoised signal. The other function is called soft-threshold:

$$
\hat{d}_{i, j}=\left\{\begin{array}{lc}
\operatorname{sgn}\left(d_{i, j}\right)\left(\left|d_{i, j}\right|-\lambda_{j}\right), & \text { for }\left|d_{i, j}\right| \geqslant \lambda_{j} \\
0, & \text { otherwise }
\end{array}\right.
$$

where $\operatorname{sgn}(\cdot)$ is sign function, which returns 1 if the element is greater than $0 ; 0$ if it equals 0 ; and -1 if it is less than 0 . 
The estimated signal is reconstructed through inverse wavelet transform on $\hat{d}_{i, j}$. It can be seen that the key point of the denoising process is selecting appropriate threshold to minimize Equation (2).

\subsection{Fruit Fly Optimization Algorithm}

FOA is a new interactive evolutionary computation method, which was proposed by Pan in 2012. By simulating the process of foraging behavior for fruit fly individuals and populations, global optimum can be obtained through appropriate iteration, as shown in Figure 1. Standard foraging process of FOA can be summarized as follows.

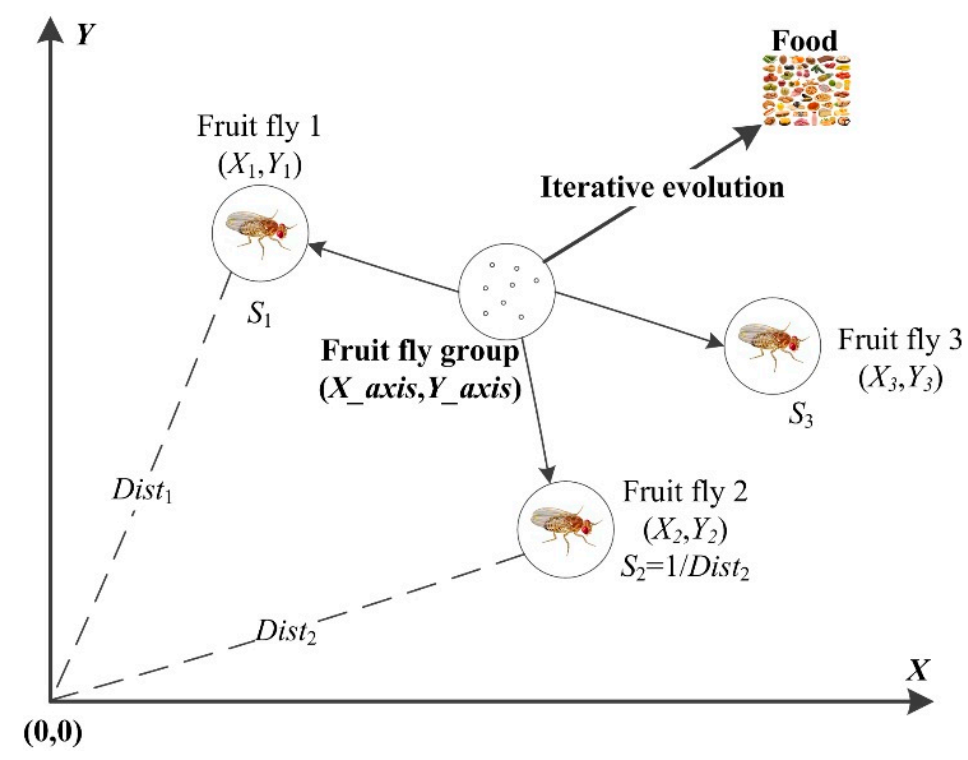

Figure 1. Process of foraging behavior for fruit fly group.

Step 1.1: The key initial parameters of the FOA are the population amount $(P A)$, the fruit fly group location range $(L R)$, the maximum iteration number $\left(I N_{\max }\right)$ and the random fly distance range $(F R)$. The initial location of the fruit fly group can be presented as follows:

$$
\left\{\begin{array}{l}
X_{-} \text {axis }=\operatorname{rand}(L R) \\
Y \_ \text {axis }=\operatorname{rand}(L R)
\end{array}\right.
$$

Step 1.2: The random direction and distance for the search of food using osphresis by an individual fruit fly is given as follows:

$$
\left\{\begin{array}{l}
X_{i}=X_{-} \text {axis }+ \text { rand }(F R) \\
Y_{i}=Y_{-} \text {axis }+\operatorname{rand}(F R)
\end{array}\right.
$$

Step 1.3: Since the food location cannot be known, the distance to the origin of coordinates $\left(\right.$ Dist $\left._{i}\right)$ and the smell concentration judgment value $\left(S_{i}\right)$ are calculated as follows:

$$
\text { Dist }_{i}=\sqrt{X_{i}^{2}+Y_{i}^{2}}, \quad S_{i}=1 / \text { Dist }_{i}
$$

The fruit fly with maximal smell concentration among the fruit fly group can be searched according to the smell concentration judgment function (or called Fitness function), which can be presented as follows:

$$
\operatorname{smell}_{i}=\text { function }\left(S_{i}\right), \quad[\text { bestsmell bestindex }]=\max (\text { smell })
$$

where bestsmell donates the maximal smell concentration, bestindex is the corresponding fruit fly number and smell is the smell concentration set of the group. 
Step 1.4: The smell concentration is compared with that of the former iteration. If it is inferior to the last generation, Steps 1.2 to 1.3 are repeated; else the best location and smell concentration can be presented as follows:

$$
\left\{\begin{array}{c}
\text { smellbest }=\text { bestsmell } \\
X \_a x i s=X(\text { bestindex }) \\
Y_{\_a x i s}=Y(\text { bestindex })
\end{array}\right.
$$

Step 1.5: When the smell concentration reaches the preset precision value or the iteration number reaches the maximal IN, the circulation stops. Otherwise, Steps 1.2 to 1.4 are repeated.

\section{The Proposed Method}

In this section, the improved fruit fly optimization algorithm is proposed to enhance the capacity of global and local research. Then the flowchart of the improved method is designed and the process of the denoising approach based on WTD-IFOA is presented.

\subsection{Improvement of FOA}

The fly distance range $(F R)$ of FOA is a random value in the range of $[-L, L]$, which can be presented as $F R \sim \mathrm{U}(-L, L)$, where $L$ named as step size. The value of $F R$ is distributed evenly among the value range. If the step size is big enough, the global search capability will be improved remarkably, while the convergence speed will be decreased obviously. Otherwise, the FOA easily gets stuck in local optimal, while has a high convergence speed.

In order to balance the global search ability and convergence rate, the distribution function of FR is modified in this paper. The value of FR follows normal distribution, $F R \sim \mathrm{N}\left(0, L^{2}\right)$. According to the characteristic of normal distribution, the probability of $F R \in[-L, L]$ is about $68.27 \%$, the probability of $F R \in[-2 L, 2 L]$ is about $95.45 \%$ and the probability of $F R \in[-3 L, 3 L]$ is about $99.73 \%$. The probability density distribution of original fly distance range and the proposed one are presented in Figure 2 . Most individuals fly towards the present best location, while more than $30 \%$ fruit flies continuing searching at a larger scale. Moreover, individuals flying towards the present optimum tend to a more concentrated region according to the probability distribution condition. Thus, the capacity of global searching and partial location are both enhanced. The flowchart of the IFOA is shown in Figure 3.
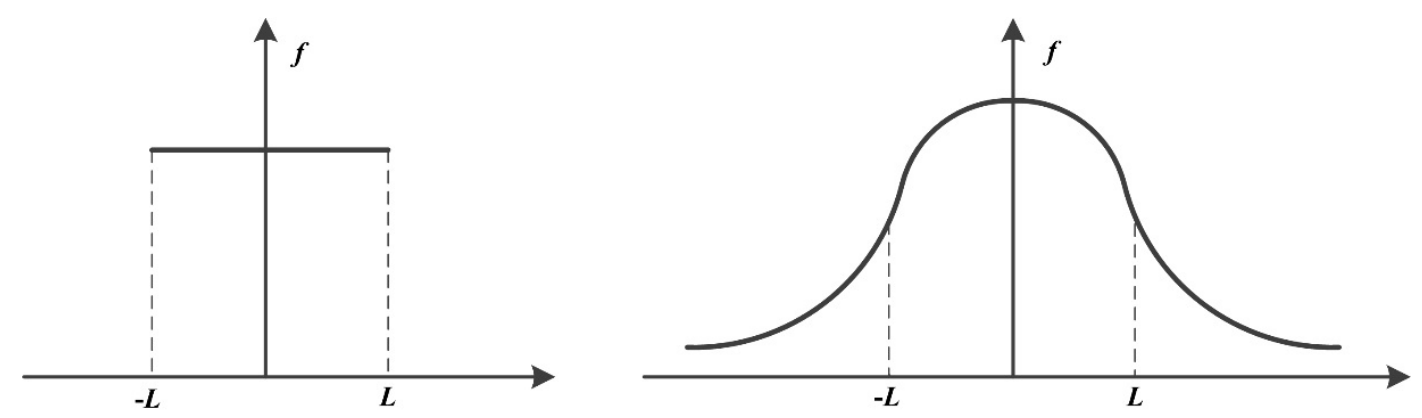

Figure 2. Probability density functions of basic fly distance range and the improved.

\subsection{Flow of the Proposed Denosing Method}

The adaptive threshold denoising method based on WTD-IFOA can be summarized as follows:

Step 2.1: For the sake of convenient calculation, the sound signal is first quantized into a certain range. Then, the initial signal is decomposed by a $J$-level wavelet transform. $i$-th coefficient in $j$-th level can be presented as $d_{i, j}$, where $i=1,2,3, \ldots, m, j=1,2,3, \ldots, J, m$ is the length of the sound signal.

Step 2.2: The parameters of IFOA are initialized, such as $L R, I N_{\max }$ and $F R$, where $F R \sim \mathrm{N}\left(0, L^{2}\right)$. For $J$-level WT, each level has an optimal threshold. So there are $J$ groups of fruit flies, each group contains $P A$ individuals. The initial location of the fruit fly group is obtained by Equation (6). 


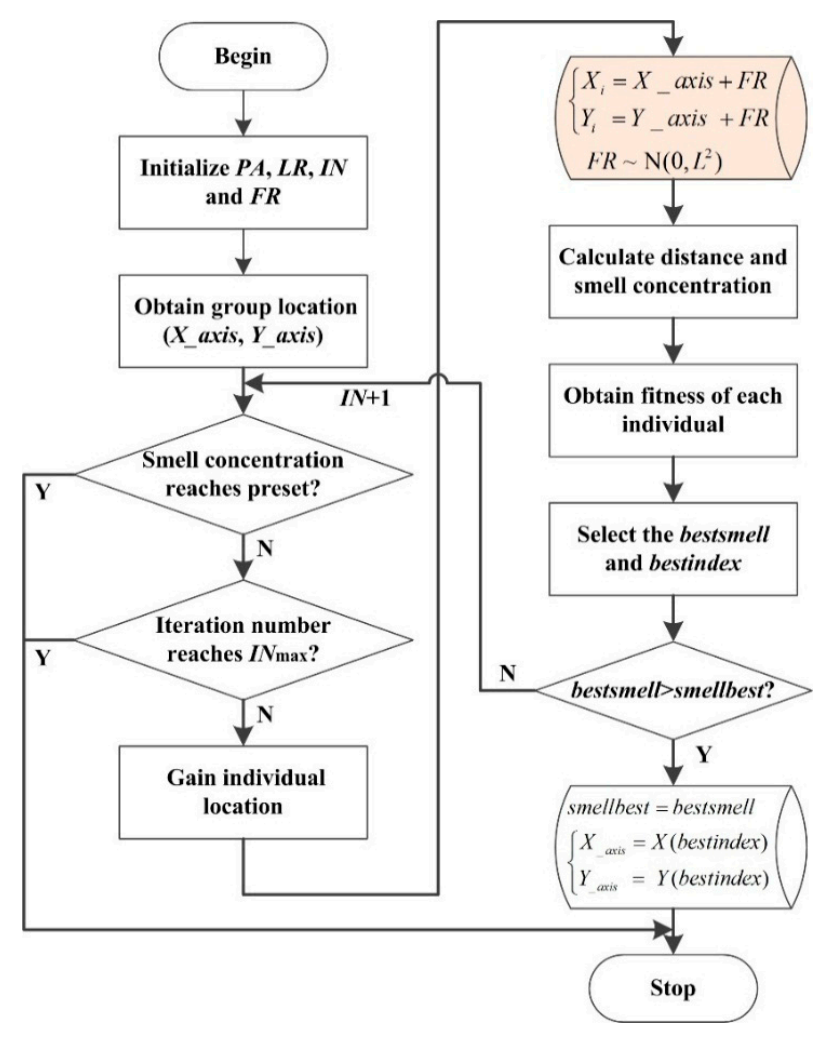

Figure 3. Flowchart of the improved fruit fly optimization algorithm.

Step 2.3: The location of each individual is gained through the fly group and $F R$. The distance and smell concentration of each fly are calculated according to Equation (8). Each smell concentration judgment is regarded as a potential threshold. Then, the useful signal and the noise component are separated according to the soft-threshold function. In order to judge the denoising performance of each fruit fly individual, the fitness function $f$ is calculated as follows:

$$
\begin{gathered}
f=\frac{1}{1+g} \\
g=\frac{\left[r_{22} \times h r_{21}\right]^{2}}{r_{11}^{2}}
\end{gathered}
$$

where $r_{22}$ is an autocorrelation of noise. As noise is yielded randomly, it has neither high autocorrelation nor zero autocorrelation. However, an ascending value implies that more original signal adheres to noise, thus the restructured signal will not be a good recovery. $h r_{21}$ is a high-order cross-correlation between useful signal and the noise. If these coefficients are descending, then it implies that both signals become more independent to each other. Thus, the original signal and noise are toward separation gradually. $r_{11}$ is an autocorrelation of useful signal. An ascending value implies that its own component is more than component of noise. Hence, the restructured signal has a good recovery [33]. $r_{22}, h r_{21}$ and $r_{11}$ are defined as follows:

$$
\begin{gathered}
r_{i j}=\frac{C_{i j}}{\sqrt{C_{i i} C_{j j}}}=\frac{\operatorname{cov}\left[s_{i}, s_{j}\right]}{\sqrt{\operatorname{cov}\left[s_{i}\right] \operatorname{cov}\left[s_{j}\right]}}, i, j=1,2 \\
h r_{i j}=\frac{H C_{i j}}{\sqrt{H C_{i i} C_{j j}}}=\frac{\operatorname{cov}\left[\varphi\left(s_{i}\right), s_{j}\right]}{\sqrt{\operatorname{cov}\left[\varphi\left(s_{i}\right)\right] \operatorname{cov}\left[s_{j}\right]}}, i, j=1,2
\end{gathered}
$$


where $s_{1}$ is the useful signal, $s_{2}$ is the noise component, $\operatorname{cov}\left[s_{i}, s_{j}\right]=\mathrm{E}\left\{\left[s_{i}-\mathrm{E}\left(s_{i}\right)\right]\left[s_{j}-\mathrm{E}\left(s_{j}\right)\right]\right\}$, $\operatorname{cov}\left[s_{i}\right]=\mathrm{E}\left\{\left[s_{i}-\mathrm{E}\left(s_{i}\right)\right]^{2}\right\}, \mathrm{E}\left(s_{i}\right)$ is mathematical expectation of $s_{i}$ and $\varphi\left(s_{i}\right)=s_{i}{ }^{2}+s_{i}{ }^{3} \cdot r_{22}, h r_{21}$ tend to minimum and $r_{11}$ tends to the maximum when the location of each fruit fly group is placed in the best. Then $g$ is the minimal and $f$ is the maximal [34].

Step 2.4: Fruit fly with maximal fitness is selected as bestsmell and the corresponding fly number is named as bestindex. If the present bestsmell is bigger than that of the former, smellbest, the corresponding coordinates are updated. Otherwise, smellbest, $X_{-a x i s}$ and $Y_{-a x i s}$ are reserved.

Step 2.5: If the ending conditions are researched, smellbest, $X_{-a x i s}$ and $Y_{\_}$axis are treated as the optimum. Otherwise, Steps 2.3 and 2.4 are repeated.

Step 2.6: The wavelet coefficients are adjusted according to the soft-threshold function and inverse wavelet transform is conducted consequently to obtain denoised signal. The flowchart of the process is shown in Figure 4.

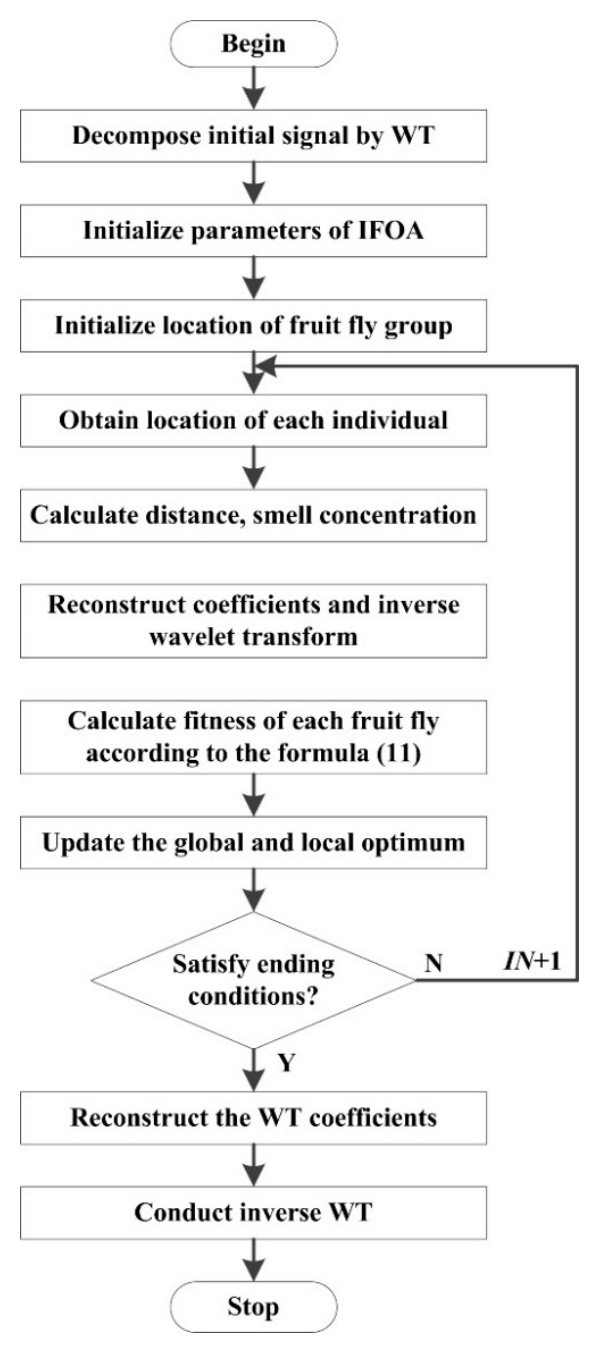

Figure 4. Process of the proposed denoising method.

\section{Simulation and Industrial Application}

In order to validate the effectiveness and superiority of the proposed method, a piece of pure sound signal of a motor was recorded. Then, Gauss white noise with different signal to noise ratio were added into the pure signal. The mean square error (MSE), peak value error (PVE) and the computation time (CT) were regarded as evaluation criteria of the noise elimination solutions. The denoising performance of standard soft threshold denoising method (SST), a threshold function-based 
noise elimination solution proposed in [10] (TFB), wavelet threshold denoising optimized by genetic algorithm (WTD-GA), wavelet threshold denoising optimized by fruit fly optimization algorithm (WTD-FOA) and the proposed WTD-IFOA were compared subsequently. Finally, an industrial application for the shearer of coal mining working face is exhibited. All calculations in this section were conducted on a workstation configured as shown in Table 1.

Table 1. Configuration of the workstation.

\begin{tabular}{cc}
\hline Operating System & Windows 7 (64 bits) \\
\hline CPU & Intel Xeon E5-2690 (8 cores, 2.9 GHz) \\
Memory & 16 GB (DDR3) \\
Hard disk space & SSD (512 GB) \\
Matlab version & 8.0 \\
\hline
\end{tabular}

\subsection{Signal Acquisition}

To test the performance of the denoising methods, a pure sound signal sequence was needed first. Because there was much background noise in industrial field, it was extremely difficult to collect the pure sound. Moreover, sound signal of a machine consisted of many frequency components, so it was also hard to synthesize a representative series with practical meaning artificially. In this paper, the sound of a motor working in a soundproof room was recorded as the original signal. Concretely, the sound signal was acquired from soundproof testing branch, Jiangsu Key Laboratory of Mining Mechanical and Electrical Equipment. The walls of the testing room were constructed of a special acoustic insulating material and echo cancellation was designed in the testing process. An AC servomotor with the rated out power of $1 \mathrm{~kW}$ and the corresponding electrical system were installed in the laboratory. The schematic of the testing room and the experiment site are shown in Figure 5.

(a)

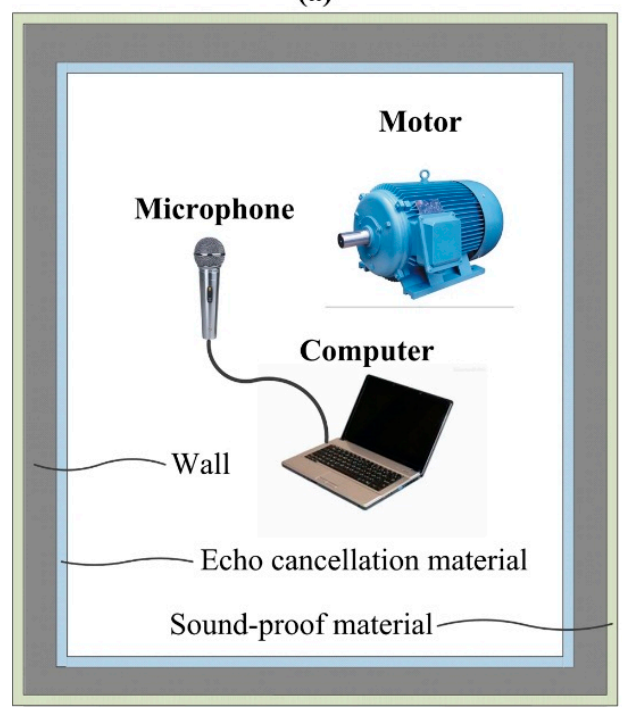

Figure 5. (a) Schematic of the soundproof testing branch; and (b) The sound recorded in site. (b)

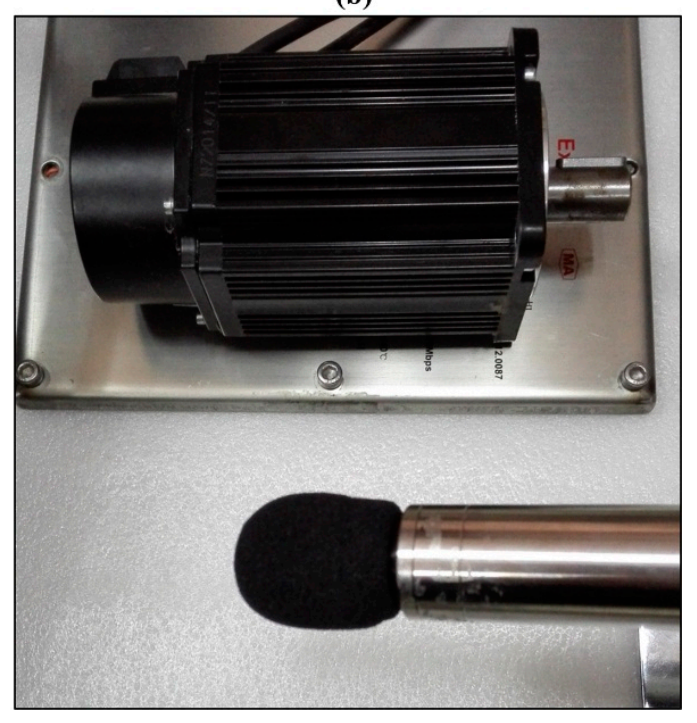

Echo cancellation material was installed on the inner wall and acoustic insulating equipment was placed on the external wall. The motor, microphone and computer were fixed in the room with the length of $6 \mathrm{~m}$, width of $5 \mathrm{~m}$ and height of $4 \mathrm{~m}$. Operators controlled the motor outside the room. The sound signal was recorded by the microphone and then transmitted to the computer. Sampling frequency of the sound signal was $44.1 \mathrm{kHz}$. The experiment was conducted as follows: start the motor remotely and keep it in no-load operation, then stop the motor after $10 \mathrm{~min}$, pretreat and save the 
sound in the computer in wav format. Quantization was subsequently operated to convert the sound amplitude into the scope of $[-1,1]$. Finally, a piece of relatively stable sound was extracted with the duration of $0.5 \mathrm{~s}$, as shown in Figure 6.

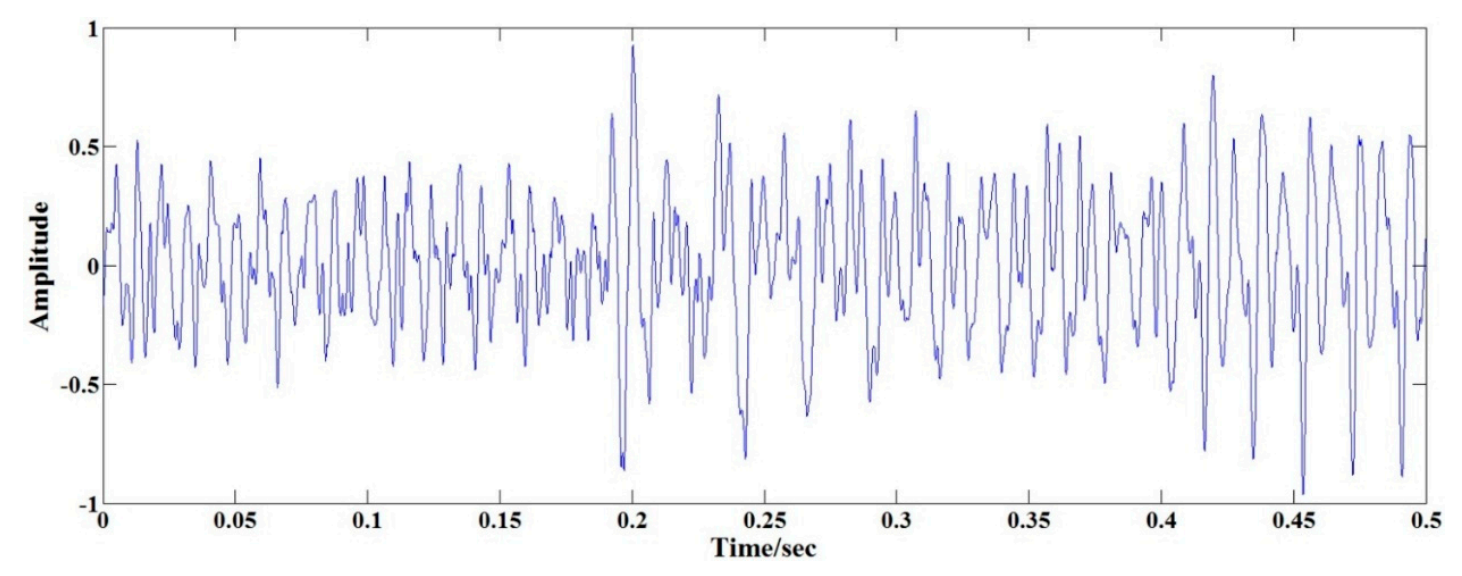

Figure 6. The extracted sound signal of the motor.

\subsection{Signal Denoising}

In this paper, the MSE $\xi$, the PVE $\eta$ and the CT $t$ were selected as evaluation indexes of the denoising methods. MSE and the PVE are calculated as follows:

$$
\begin{gathered}
\xi=\frac{1}{k} \sum_{i=1}^{k}\left[d s_{i}-s_{i}\right]^{2} \\
\eta=\frac{\left|P_{o}-P_{d}\right|}{P_{o}} \times 100 \%
\end{gathered}
$$

where $d s$ is denoised signal, $s$ is original signal, $k$ is the length of the signal, and $P_{o}$ and $P_{d}$ are, respectively, the peak value of the original signal and denoised signal. To test the performance of SST, TFB, WTD-GA, WTD-FOA and the proposed WTD-IFAO, Gauss white noise was added into the original sound. The signal to noise ratio (SNR) $\zeta(\mathrm{dB})$ was introduced to measure the degree of noise, and SNR was defined as follows:

$$
\zeta=10 \lg \frac{\sum_{i=1}^{k} s_{i}^{2}}{\sum_{i=1}^{k} n_{i}^{2}}
$$

where $s_{i}$ was the amplitude of the original signal and $n_{i}$ was that of the added noise.

The denoising process was conducted as follows:

(1) Add Gaussian white noise into the original sound and conduct wavelet decomposition. A noisy signal with $\zeta=5 \mathrm{~dB}$ was firstly analyzed and the synthesis was finished in Matlab 8.0 (MathWorks Inc., Natick, MA, USA, 2012). Then the synthetic signal was decomposed by wavelet decomposition with db2 wavelet at 5 levels [6]. The decomposition result is shown in Figure 7.

(2) Denoise the noisy signal by SST. The value range of the wavelet threshold was firstly calculated according to Equation (3). The recommended threshold $\lambda_{\text {rec }}$ was adopted according to Donoho, where $q=0.6745$. The wavelet coefficients of each level were shrunk according to Equation (5). Then the signal was reconstructed by inverse WT. 
(3) Denoise the noisy signal by TFB. An improved threshold function with continuous first and second order derivative was introduced in [10], and is presented as follows:

$$
\hat{d}_{i, j}=\left\{\begin{array}{cc}
d_{i, j}+\lambda-\frac{\lambda}{2 k+1}, & d_{i, j}<-\lambda \\
\frac{1}{(2 k+1) \lambda^{2 k}} \cdot d_{i, j} 2 k+1, & \left|d_{i, j}\right|<\lambda \\
d_{i, j}-\lambda+\frac{\lambda}{2 k+1}, & d_{i, j}>\lambda
\end{array}\right\}
$$

where $\lambda$ is determined according to Equation (3) and $k=3$.

(4) Denoise the noisy signal by WTD-GA. The maximum $\lambda_{\max }$ appeared at $q=1$ and $\lambda_{\min }$ obtained when $q=0.4$, so $\lambda \in\left[\lambda_{\min }, \lambda_{\max }\right]$. The population size was 100 , each chromosome was a five-dimensional vector, the crossover probability was 0.7 , the mutation probability was 0.01 and the most iteration generation was 100, as recommended by ref. [15].

(5) Denoise the noisy signal by WTD-FOA. The parameters were set as follows: $\lambda \in\left[\lambda_{\min }, \lambda_{\max }\right]$. $\lambda_{\min }$ and $\lambda_{\max }$ were calculated as previously. The fly distance obeys uniform distribution, $F R \sim \mathrm{U}(-0.2,0.2)$. The population number was 5 , each group contained 20 individuals and the iteration number was 100. The fitness of each fruit fly was calculated according to Equation (11).

(6) Denoise the noisy signal by WTD-IFOA. The parameters were set as follows: $\lambda \in\left[\lambda_{\text {min }}, \lambda_{\text {max }}\right]$, the fly distance obeys normal distribution, $F R \sim \mathrm{N}\left(0,0.2^{2}\right)$. The population number was 5 , each group contained 20 individuals and the iteration number was 100 . The fitness of each fruit fly was calculated according to Equation (11).

Subsequently, a comprehensive comparison was made for the five methods. The $\xi, \eta$ and $t$ at $\zeta=5 \mathrm{~dB}$ of the average value of the five simulation results are presented in Table 2 . And the denoised signals of the 5 solutions were shown in Figure 8. It can be seen in the table that adaptive denoising methods based on intelligent optimization had a better comprehensive performance than SST and TFB. MSE of the WTD-FOA was smaller than that of WTD-GA while the PVE was contrary, which indicated the two methods had no optimal solution, both in global and local. The WTD-IFOA overcame the disadvantage and contributed a superior scheme. The MSE of WTD-IFOA was decreased about $35.36 \%$ compared with SST, and the PVE decreased about $9.40 \%$. As optimal threshold was obtained through the iterative process, the last four methods were much more time-consuming. Among these approaches, TFB cost the most time due to its complex calculation. The optimization process of WTD-GA was much more complex than the other intelligent solutions from the table. Moreover, the WTD-IFOA, respectively, saved $26.96 \%$ and $12.47 \%$ time compared with WTD-GA and WTD-FOA because of its stronger addressing ability.

Table 2. Comparison of the five methods $(\mathrm{SNR}=5 \mathrm{~dB})$.

\begin{tabular}{cccccc}
\hline Method & SST & TFB & WTD-GA & WTD-FOA & WTD-IFOA \\
\hline MSE $\left(\times 10^{-4}\right)$ & 12.19 & 9.61 & 11.78 & 8.53 & 7.88 \\
PVE $(\%)$ & 10.56 & 7.39 & 2.18 & 2.28 & 1.16 \\
CT (s) & 0.87 & 24.61 & 11.24 & 9.38 & 8.21 \\
\hline
\end{tabular}

In order to research the denoising performance at different SNR, a further comparison was made and the average value of five simulation results are shown in Figure 9. Four noisy signal with $\mathrm{SNR}=5 \mathrm{~dB}, 10 \mathrm{~dB}, 15 \mathrm{~dB}$ and $20 \mathrm{~dB}$ were synthetized and handled. Then the MSE, PVE and CT of the denoising processes were presented in the figure. It can be roughly obtained that the three evaluation parameters decreased with the SNR. As the threshold value of each level and wavelet coefficients were determined directly by Equations (3) and (4) in SST, the denoising process could be finished in a short time, while the TFB based on gradient descent algorithm was time-consuming in different SNR as its complex calculation process. Denoising methods using optimization algorithm had a comprehensive denoising performance. The computation time was decreased sharply compared with TFB while 
it still cost more time than SST. In detail, the WTD-GA did not obviously vary from WTD-FOA in PVE, while it had a distinct weakness in MSE and CT compared with FOA-based methods. It could be seen from the simulation results that the TFB, WTD-GA and WTD-FOA fell into local extreme during the parameters optimization. Moreover, the WTD-IFOA exhibited obvious superiority in the denoising effect, which revealed its terrific global and local ability compared to the other methods in different SNR.

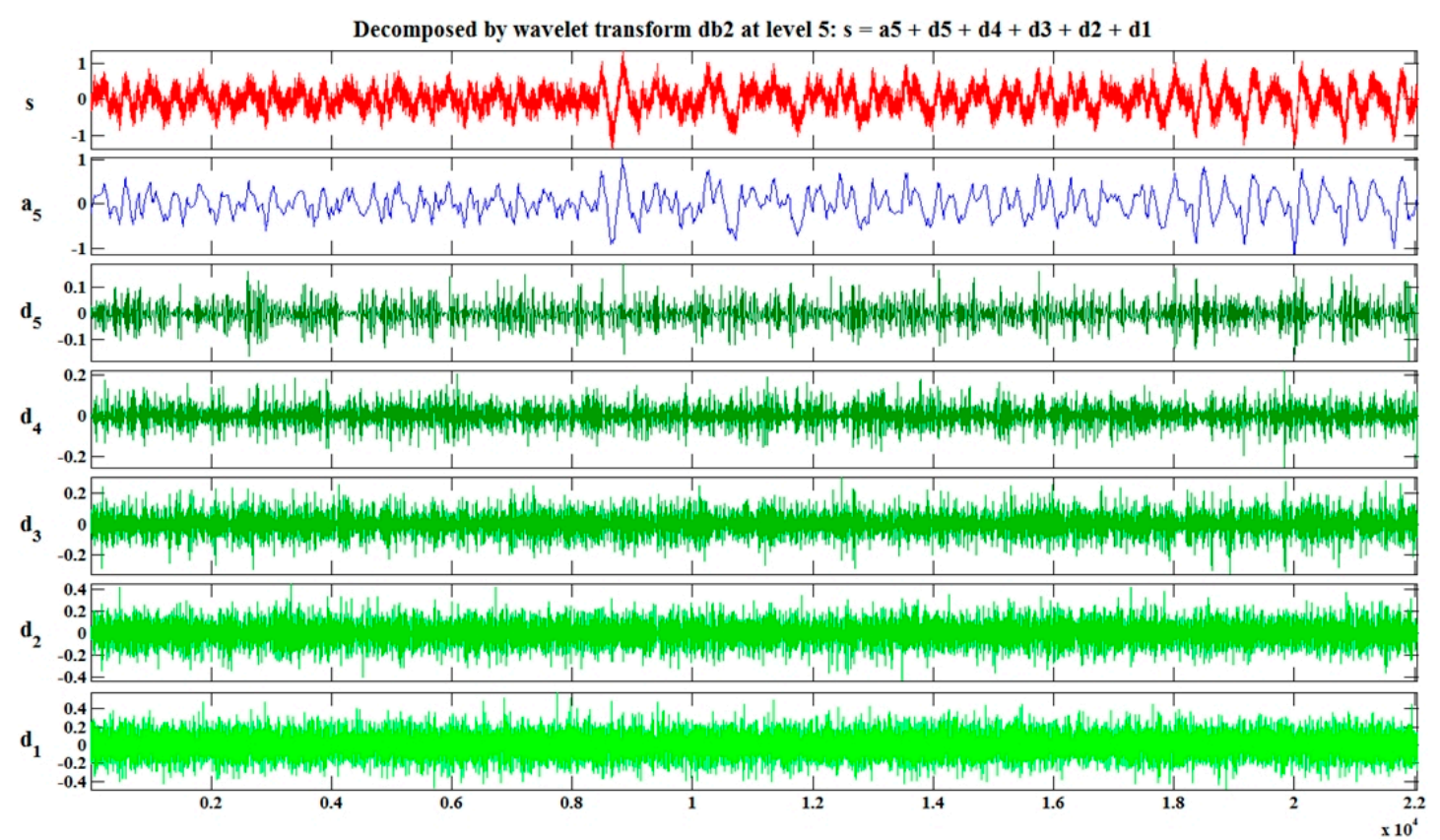

Figure 7. Gauss white noise-added signal and its wavelet coefficients (SNR $=5 \mathrm{~dB})$.
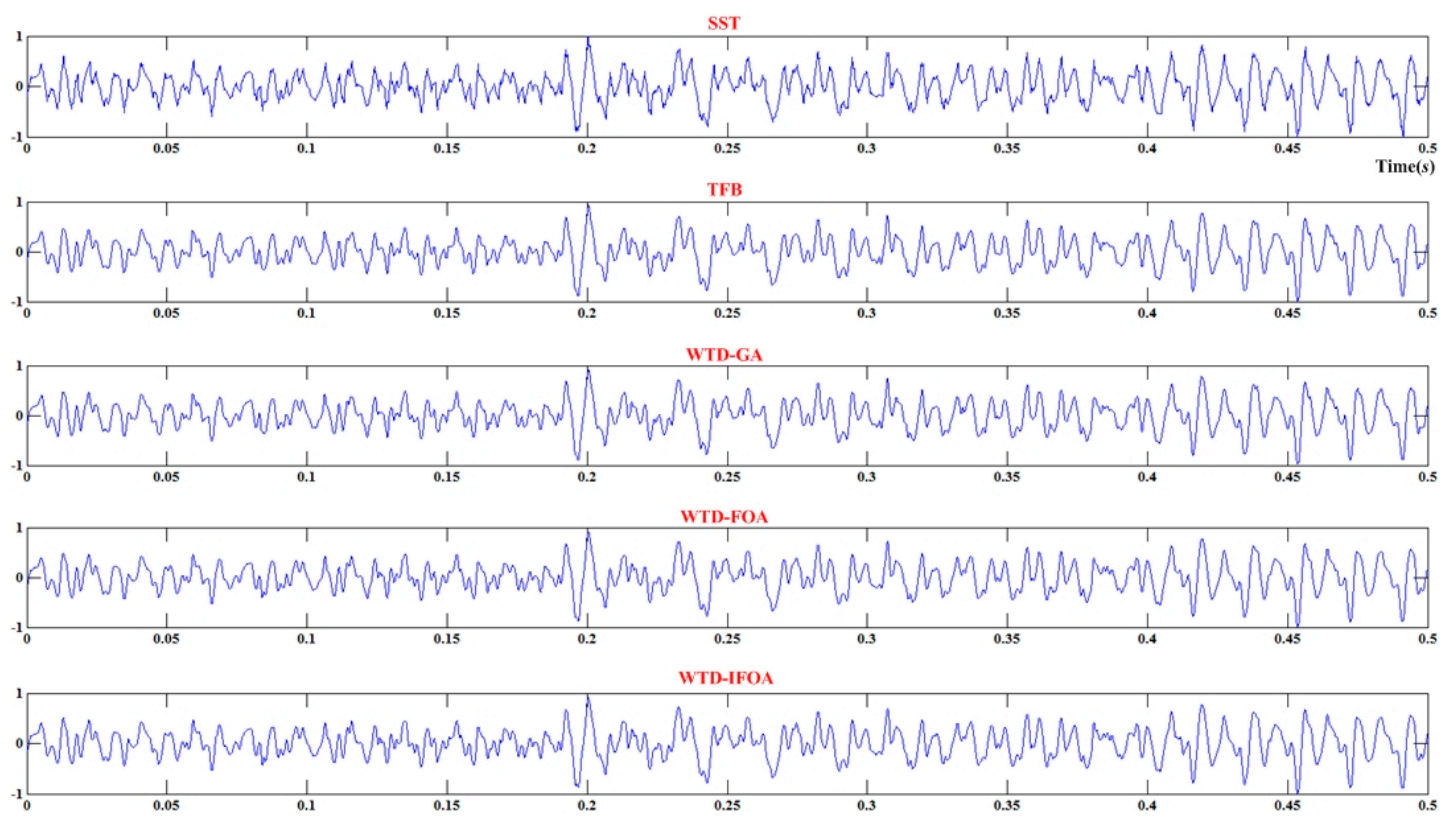

Figure 8. Denoise result of the five methods (SNR $=5 \mathrm{~dB})$. 

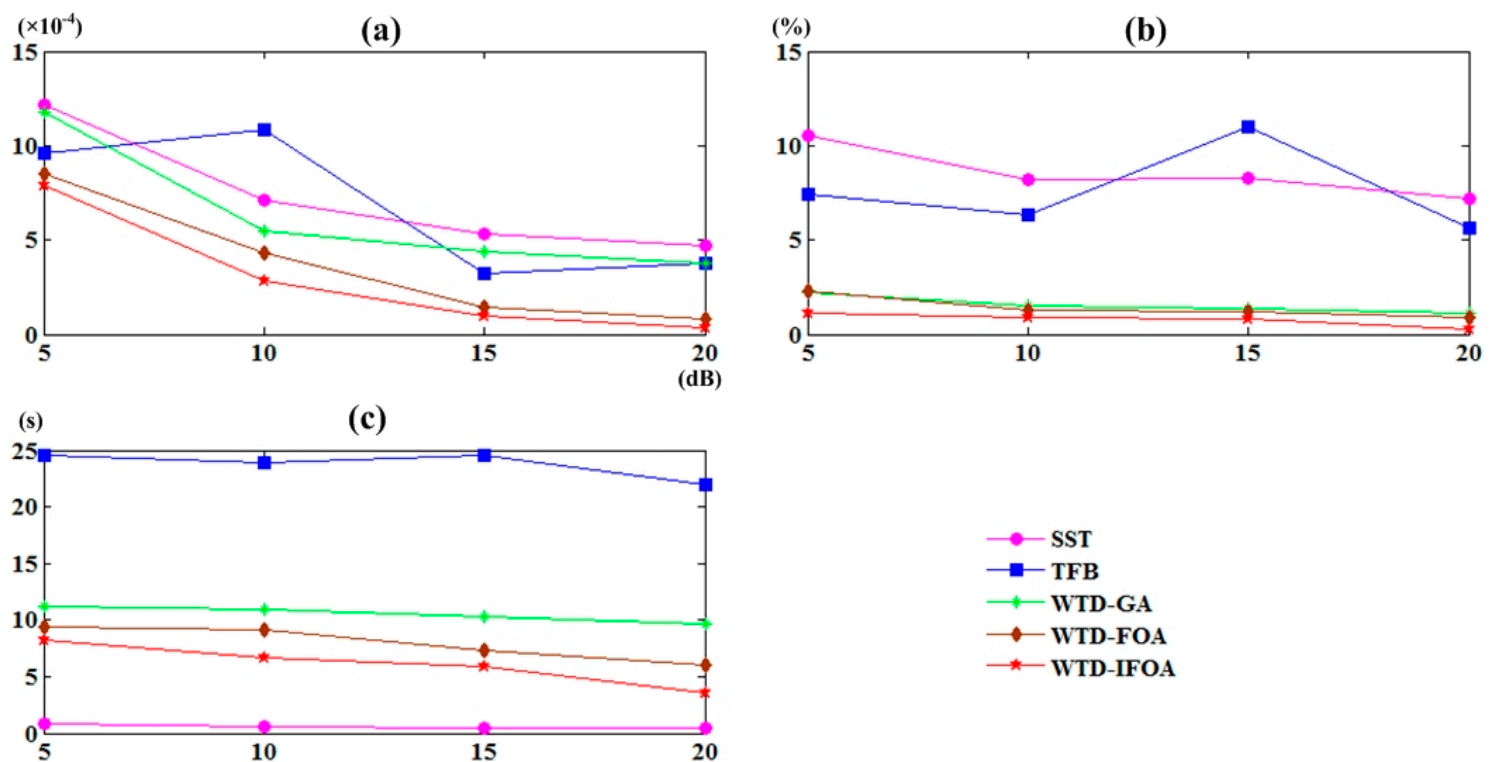

Figure 9. Comprehensive comparison of the five methods: (a) Mean square error of the five methods; (b) Peak value error of the five methods; and (c) Computation time of the five methods.

\subsection{Application}

In order to test practical effect of the proposed adaptive denoising method for the machinery sound signal based on WTD-IFOA, an industrial application was operated in a fully-mechanized coal mining working face. The shearer is an important machine in automatic coal mining, and working condition monitoring for the shearer is of great necessity. Traditional monitoring methods are mainly based on the vibration signal [35], even though the working life of the vibration sensors are very short due to the bad working condition and contact-measurement. Coal output is seriously restricted by the frequent maintenance. In the August 2015, an online monitoring system through the shearer cutting sound signal was built in the 71,507 coal mining face in the NO.2 Mine of Yangquan Coal Industry Group Corporation. However, there existed a large number of noise signal among the initial signal because of the harsh working environment. To eliminate the background noise from the sound, an industrial microphone was installed and WTD-IFOA was applied. The three-dimensional model of the coal mining shearer and field sound collection is presented in Figure 10.
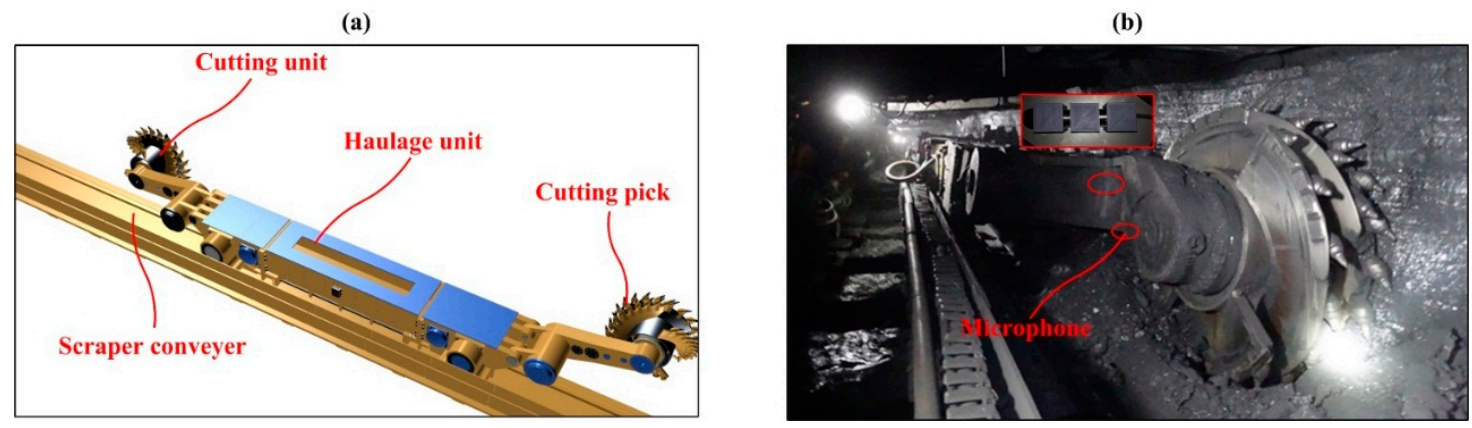

Figure 10. (a) Three-dimensional model of the shearer; and (b) collection of field cutting sound.

To illustrate the effectiveness of the proposed method, a piece of field sound signal with the length of $0.5 \mathrm{~s}$ was extracted and denoised. The original sound and the denoised one are shown in Figure 11. Then, 4096 points FFT was conducted to analyze the frequency components of the two signals, as presented in Figure 12. It can be seen in Figure 12 that the processed signal was sharp 
decreased in amplitude compared with the original field signal. The reason lies in that the scope saltation caused by the noise component was removed. Moreover, frequency components of original signal shown in Figure 12 had a disordered distribution, and it was difficult to identify the working state of the shearer. On the contrary, it was regular for the denoised signal. Some wave peaks appeared in the spectrogram, and other areas were stable. Different spectra of the collected signal reflected different working conditions of the shearer. Thus, the working state could be identified according to the wave peaks.

(a)

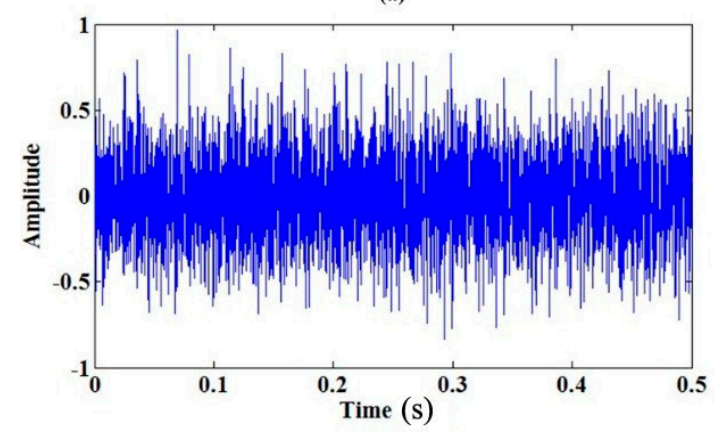

(b)

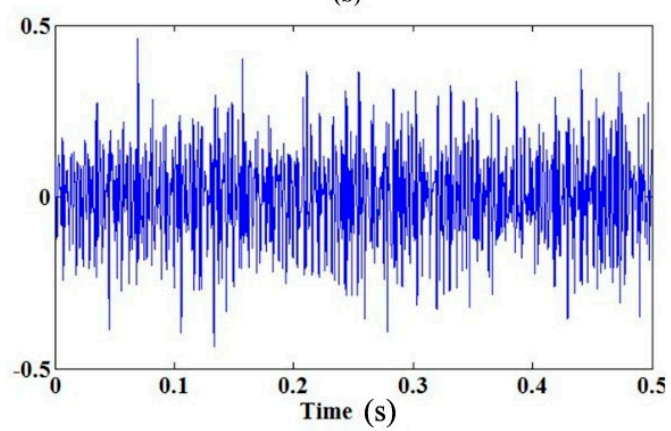

Figure 11. (a) The orginal sound signal; and (b) the denoised sound signal.

(a)

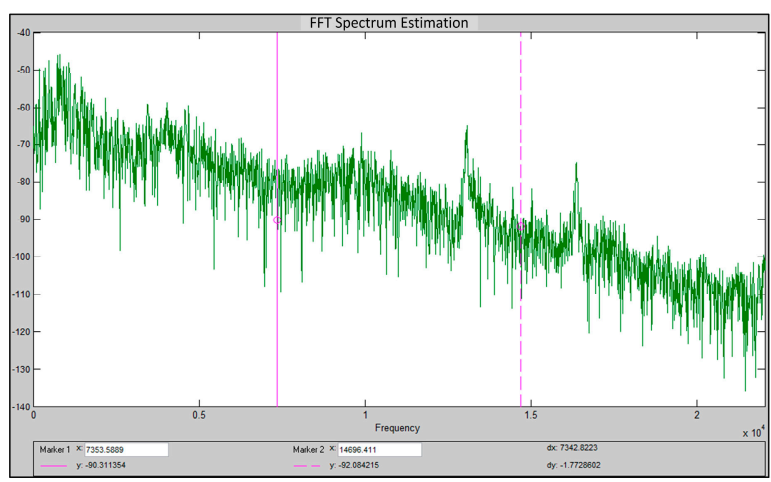

(b)

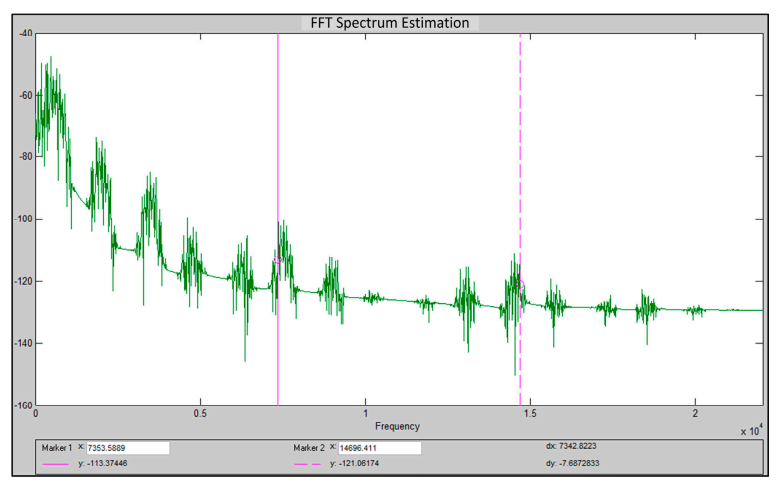

Figure 12. (a) Frequency components of the original signal; and (b) frequency components of the denoised signal.

\section{Conclusions and Future Work}

In order to eliminate noise components from the sound signal of a working machine, this paper proposes a novel approach based on wavelet threshold denoising and an improved FOA. Improved strategy on the basis of fly distance range obeying normal distribution was applied in the denoising process. To verify the feasibility and superiority of the proposed WTD-IFOA, a simulation example was provided and some comparisons were conducted. The simulation example and comparison results showed that the adaptive denoising method could effectively eliminate noise components and the proposed approach outperformed the others. Finally, an industrial application was performed on the shearer in a fully-mechanized coal mining face to test practical effect.

However, there are also some deficiencies and shortcomings in this method including the following. On the one hand, although the proposed WTD-IFOA is much more timesaving than other adaptive denoising approaches, the calculation duration is still a serious problem that cannot be neglected. On the other hand, parameters during the optimization process are determined according to past scholars and a large number of simulation experiments, while strict mathematical deduction are lacking. In future studies, the authors plan to investigate some improvements to the proposed 
approach. These may include an improved algorithm code with higher execution efficiency and appropriate scheme for determining optimization parameters.

Acknowledgments: The support of Joint Funds of the National Natural Science Foundation of China (No. U1510117), National Key Basic Research Program of China (No. 2014CB046301) and the Priority Academic Program Development (PAPD) of Jiangsu Higher Education Institutions in carrying out this research are gratefully acknowledged.

Author Contributions: Zhongbin Wang and Jing Xu contributed the new processing method; Chao Tan and Lin Zhang designed the simulations and experiments; Lei Si and Xinhua Liu performed the experiments; and Jing $\mathrm{Xu}$ wrote the paper.

Conflicts of Interest: The authors declare no conflict of interest.

\section{References}

1. Lin, J.; Qu, L.S. Feature extraction based on Morlet wavelet and its application for mechanical fault diagnosis. J. Sound Vib. 2000, 234, 135-148. [CrossRef]

2. $\quad \mathrm{Li}, \mathrm{X}$.; Bassluny, A.M. Transient dynamical analysis of strain signals in sheet metal stamping processes. Int. J. Mach. Tool. Man. 2008, 48, 576-588. [CrossRef]

3. Xue, W.F.; Chen, J.; Li, J.Q.; Liu, X.F. Acoustical feature extraction of rotating machinery with combined wave superposition and blind source separation. Proc. Inst. Mech. Eng. C J. Mech. Eng. Sci. 2006, 220, 1423-1431. [CrossRef]

4. Benko, U.; Petrovcic, J.; Juricic, D.; Tavcarb, J.; Rejec, J. An approach to fault diagnosis of vacuum cleaner motors based on sound analysis. Mech. Syst. Signal Process. 2005, 19, 427-445. [CrossRef]

5. Ning, D.Y.; Gong, Y.J. Shocking fault component of abnormal sound signal in the fault engine extract method based on linear superposition method and cross-correlation analysis. Adv. Mech. Eng. 2015, 7. [CrossRef]

6. Abbasiona, S.; Rafsanjania, A.; Farshidianfarb, A.; Iranic, N. Rolling element bearings multi-fault classification based on the wavelet denoising and support vector machine. Mech. Syst. Signal Process. 2007, 21, 2933-2945. [CrossRef]

7. White, D.J.; William, P.E.; Hoffman, M.W.; Balkir, S. Low-Power Analog Processing for Sensing Applications: Low-Frequency Harmonic Signal Classification. Sensors 2013, 13, 9604-9623. [CrossRef] [PubMed]

8. Mallat, F.G. A Theory for Multiresolution Signal Decomposition: The Wavelet Representation. IEEE Trans. Pattern Anal. 1989, 11, 674-693. [CrossRef]

9. Donoho, D.L. De-Noising by Soft-Thresholding. IEEE Trans. Inform. Theory 1995, 41, 613-627. [CrossRef]

10. Zhang, X.P.; Desai, M.D. Adaptive Denoising Based on SURE Risk. IEEE Signal Proc. Lett. 1998, 5, 265-267. [CrossRef]

11. Nasri, M.; Nezamabadi-pour, H. Image denoising in the wavelet domain using a new adaptive thresholding function. Neurocomputing 2009, 72, 1012-1025. [CrossRef]

12. Zhang, B.; Sun, L.X.; Yu, H.B.; Xin, Y.; Cong, Z.B. A method for improving wavelet threshold denoising in laser-induced breakdown spectroscopy. Spectrochim. Acta B 2015, 107, 32-44. [CrossRef]

13. Tian, J.; Yu, W.Y.; Ma, L.H. AntShrink: Ant colony optimization for image shrinkage. Pattern Recognit. Lett. 2010, 31, 1751-1758. [CrossRef]

14. Bhutada, G.G.; Anand, R.S.; Saxena, S.C. PSO-based learning of sub-band adaptive thresholding function for image denoising. Signal Image Video Proc. 2012, 6, 1-7. [CrossRef]

15. Li, J.; Cheng, C.; Jiang, T.; Grzybowski, S. Wavelet De-noising of Partial Discharge Signals Based on Genetic Adaptive Threshold Estimation. IEEE Trans. Dielectr. Electr. Insul. 2012, 19, 543-549.

16. Pan, W.T. A new Fruit Fly Optimization Algorithm: Taking the financial distress model as an example. Knowl. Based Syst. 2012, 26, 69-74. [CrossRef]

17. Pan, W.-T. Using modified fruit fly optimisation algorithm to perform the function test and case studies. Connect. Sci. 2013, 25, 151-160. [CrossRef]

18. Pan, W.-T. Mixed modified fruit fly optimization algorithm with general regression neural network to build oil and gold prices forecasting model. Kybernetes 2014, 43, 1053-1063. [CrossRef]

19. Xu, W.J.; Deng, X.; Li, J. A New Fuzzy Portfolio Model Based on Background Risk Using MCFOA. Int. J. Fuzzy Syst. 2015, 17, 246-255. [CrossRef] 
20. Xing, Y.F. Design and optimization of key control characteristics based on improved fruit fly optimization algorithm. Kybernetes 2013, 42, 466-481. [CrossRef]

21. Lin, S.M. Analysis of service satisfaction in web auction logistics service using a combination of Fruit fly optimization algorithm and general regression neural network. Neural Comput. Appl. 2013, 22, 783-791. [CrossRef]

22. Wang, W.C.; Liu, X.G. Melt index prediction by least squares support vector machines with an adaptive mutation fruitfly optimization algorithm. Chemometr. Intell. Lab. 2015, 141, 79-87. [CrossRef]

23. Wang, L.; Shi, Y.L.; Liu, S. An improved fruit fly optimization algorithm and its application to joint replenishment problems. Expert Syst. Appl. 2015, 42, 4310-4323. [CrossRef]

24. Pan, Q.K.; Sang, H.Y.; Duan, J.H.; Gao, L. An improved fruit fly optimization algorithm for continuous function optimization problems. Knowl. Based Syst. 2014, 62, 69-83. [CrossRef]

25. Donoho, D.L.; Johnstone, I.M. Adapting to unknown smoothness via wavelet shrinkage. J. Am. Stat. Assoc. 1995, 90, 1200-1224. [CrossRef]

26. Yi, T.H.; Li, H.N.; Zhao, X.Y. Noise Smoothing for Structural Vibration Test Signals Using an Improved Wavelet Thresholding Technique. Sensors 2012, 12, 11205-11220. [CrossRef] [PubMed]

27. Meng, B.; Li, Z.P.; Wang, H.H.; Li, Q.S. An improved wavelet adaptive logarithmic threshold denoising method for analysing pressure signals in a transonic compressor. Proc. Inst. Mech. Eng. C J. Mech. Eng. Sci. 2015, 229, 2023-2030. [CrossRef]

28. Soni, V.; Bhandari, A.K.; Kumar, A.; Singh, G.K. Improved sub-band adaptive thresholding function for denoising of satellite image based on evolutionary algorithms. IET Signal Process. 2013, 7, 720-730. [CrossRef]

29. Li, H.Z.; Guo, S.; Zhao, H.R.; Su, C.B.; Wang, B. Annual Electric Load Forecasting by a Least Squares Support Vector Machine with a Fruit Fly Optimization Algorithm. Energies 2012, 5, 4430-4445. [CrossRef]

30. Ramachandran, B.; Bellarmine, G.T. Improving observability using optimal placement of phasor measurement units. Int. J. Elec. Power 2014, 56, 55-63. [CrossRef]

31. Yuan, X.F.; Dai, X.S.; Zhao, J.Y.; He, Q. On a novel multi-swarm fruit fly optimization algorithm and its application. Appl. Math. Comput. 2014, 233, 260-271. [CrossRef]

32. Shan, D.; Cao, G.H.; Dong, H.J. LGMS-FOA: An Improved Fruit Fly Optimization Algorithm for Solving Optimization Problems. Math. Probl. Eng. 2013. [CrossRef]

33. Liu, C.C.; Sun, T.Y.; Tsai, S.J.; Yu, Y.H.; Hsieh, S.T. Heuristic wavelet shrinkage for denoising. Appl. Soft Comput. 2011, 2011, 256-264. [CrossRef]

34. Lou, S.T.; Zhang, X.D. Fuzzy-based learning rate determination for blind source separation. IEEE Trans. Fuzzy Syst. 2003, 11, 375-383.

35. Zhao, L.J.; Tian, Z. Vibration characteristics of thin coal seam shearer. Chin. J. Vib. Shock 2015, 34, 195-199.

(C) 2016 by the authors; licensee MDPI, Basel, Switzerland. This article is an open access article distributed under the terms and conditions of the Creative Commons Attribution (CC-BY) license (http:/ / creativecommons.org/licenses/by/4.0/). 